PROCEEDINGS OF THE

AMERICAN MATHEMATICAL SOCIETY

Volume 131, Number 5, Pages 1415-1422

S 0002-9939(02)06682-0

Article electronically published on September 5, 2002

\title{
LINEAR PERTURBATIONS OF A NONOSCILLATORY SECOND ORDER DIFFERENTIAL EQUATION II
}

\author{
WILLIAM F. TRENCH
}

(Communicated by Carmen C. Chicone)

\begin{abstract}
Let $y_{1}$ and $y_{2}$ be principal and nonprincipal solutions of the nonoscillatory differential equation $\left(r(t) y^{\prime}\right)^{\prime}+f(t) y=0$. In an earlier paper we showed that if $\int^{\infty}(f-g) y_{1} y_{2} d t$ converges (perhaps conditionally), and a related improper integral converges absolutely and sufficently rapidly, then the differential equation $\left(r(t) x^{\prime}\right)^{\prime}+g(t) x=0$ has solutions $x_{1}$ and $x_{2}$ that behave asymptotically like $y_{1}$ and $y_{2}$. Here we consider the case where $\int^{\infty}(f-g) y_{2}^{2} d t$ converges (perhaps conditionally) without any additional assumption requiring absolute convergence.
\end{abstract}

\section{INTRODUCTION}

We consider the differential equation

$$
\left(r(t) x^{\prime}\right)^{\prime}+g(t) x=0
$$

as a perturbation of

$$
\left(r(t) y^{\prime}\right)^{\prime}+f(t) y=0,
$$

under the following standing assumption.

Assumption A. Let $r$ and $f$ be real-valued and continuous, with $r>0$, on $[a, \infty)$. Suppose that (2) is nonoscillatory at infinity. Let $g$ be continuous and possibly complex-valued on $[a, \infty)$.

It is known [4, p. 355] that since (2) is nonoscillatory at infinity, it has solutions $y_{1}$ and $y_{2}$ which are positive on $[b, \infty)$ for some $b \geq a$ and satisfy the following conditions:

$$
\begin{gathered}
r\left(y_{1} y_{2}^{\prime}-y_{1}^{\prime} y_{2}\right)=1, \quad t \geq a, \\
\lim _{t \rightarrow \infty} \frac{y_{2}(t)}{y_{1}(t)}=\infty .
\end{gathered}
$$

Without loss of generality we let $b=a$. Henceforth $t \geq a$. It is convenient to define

$$
\rho=y_{2} / y_{1}
$$

Received by the editors July 10, 2001 and, in revised form, December 6, 2001. 2000 Mathematics Subject Classification. Primary 34A30.

Key words and phrases. Asymptotic, nonoscillatory, principal, nonprincipal. 
From (3) and (4),

$$
\rho^{\prime}=1 / r y_{1}^{2}>0 \quad \text { and } \quad \lim _{t \rightarrow \infty} \rho(t)=\infty .
$$

We use the Landau symbols " $O$ " and "O" in the standard way to denote behavior as $t \rightarrow \infty$. In [6] we proved the following theorem.

Theorem 1. Suppose that $\int^{\infty}(f-g) y_{1} y_{2} d t$ converges (perhaps conditionally) and

$$
\sup _{\tau \geq t}\left|\int_{\tau}^{\infty}(f-g) y_{1} y_{2} d s\right| \leq \phi(t)
$$

where $\phi(t) \rightarrow 0$ monotonically as $t \rightarrow \infty$. Define

$$
G(t)=\int_{t}^{\infty}(f-g) y_{1}^{2} d s
$$

and suppose that

$$
\int^{\infty}|G| \phi \rho^{\prime} d t<\infty
$$

and

$$
\limsup _{t \rightarrow \infty}(\phi(t))^{-1} \int_{t}^{\infty}|G| \phi \rho^{\prime} d s=A<1 / 3
$$

Then (1) has a solution $x_{1}$ such that

$$
x_{1}=y_{1}(1+O(\phi))
$$

and

$$
\left(x_{1} / y_{1}\right)^{\prime}=O\left(\phi \rho^{\prime} / \rho\right),
$$

and a solution $x_{2}$ such that

$$
x_{2}=y_{2}\left(1+O\left(\phi_{m}\right)\right)
$$

and

$$
\left(x_{2} / y_{2}\right)^{\prime}=O\left(\phi_{m} \rho^{\prime} / \rho\right),
$$

where

$$
\phi_{m}=\max \{\phi, \hat{\phi}\}
$$

with

$$
\hat{\phi}(t)=\frac{1}{\rho(t)} \int_{a}^{t} \rho^{\prime} \phi d s .
$$

This result was an improvement on a theorem of Hartman and Wintner [4, p. 379], and it was subsequently improved by Chen [1] and Šimša [5]. (For more on the Hartman-Wintner problem, see 2 and [3].) In this continuation of [6] we consider the case where $\int^{\infty}(f-g) y_{2}^{2} d t$ converges, perhaps conditionally. To motivate the present work, we first apply Theorem 1 under this assumption.

Let

$$
H(t)=\int_{t}^{\infty}(f-g) y_{1} y_{2} d s
$$

and recall from (7) that

$$
\sup _{\tau \geq t}\{|H(\tau)|\} \leq \phi(t) .
$$


Let

$$
I(t)=\int_{t}^{\infty}(f-g) y_{2}^{2} d s
$$

and suppose that

$$
\sup _{\tau \geq t}\{|I(\tau)|\} \leq \sigma(t)
$$

where $\sigma(t) \rightarrow 0$ monotonically as $t \rightarrow \infty$. From (8), (10), and (11),

$$
H(t)=-\int_{t}^{\infty} \frac{I^{\prime}}{\rho} d s=\frac{I(t)}{\rho(t)}+\int_{t}^{\infty} I\left(\frac{1}{\rho}\right)^{\prime} d s
$$

and

So

$$
G(t)=-\int_{t}^{\infty} \frac{I^{\prime}}{\rho^{2}} d s=\frac{I(t)}{\rho^{2}(t)}+\int_{t}^{\infty} I\left(\frac{1}{\rho^{2}}\right)^{\prime} d s
$$

$$
|H(t)| \leq 2 \sigma(t) / \rho(t) \quad \text { and } \quad|G(t)| \leq 2 \sigma(t) / \rho^{2}(t)
$$

It is straightforward to verify that (9) holds with $\phi=\sigma / \rho$ and $A=0$. Therefore Theorem 1 implies that (1) has solutions $x_{1}$ and $x_{2}$ such that

$$
\begin{gathered}
x_{1}=y_{1}(1+O(\sigma / \rho)), \\
\left(x_{1} / y_{1}\right)^{\prime}=O\left(\sigma \rho^{\prime} / \rho^{2}\right), \\
x_{2}=y_{2}(1+O(\hat{\phi})),
\end{gathered}
$$

and

$$
\left(x_{2} / y_{2}\right)^{\prime}=O\left(\hat{\phi} \rho^{\prime} / \rho\right)
$$

with

$$
\hat{\phi}(t)=\frac{1}{\rho(t)} \int_{a}^{t} \frac{\sigma \rho^{\prime}}{\rho} d s .
$$

At best, (17) and (18) imply that

$$
x_{2}=y_{2}(1+O(1 / \rho))
$$

and

$$
\left(x_{2} / y_{2}\right)^{\prime}=O\left(\rho^{\prime} / \rho^{2}\right)
$$

if $\int_{a}^{\infty} \sigma \rho^{\prime} / \rho d s<\infty$, which may be false. Among other things, we will show that (17) and (18) can be replaced by

$$
x_{2}=y_{2}(1+O(\sigma / \rho))
$$

and

$$
\left(x_{2} / y_{2}\right)^{\prime}=O\left(\sigma \rho^{\prime} / \rho^{2}\right) .
$$

These two equations are improvements over (17) and (18), since $\lim _{t \rightarrow \infty} \rho(t) \hat{\phi}(t) / \sigma(t)$ $=\infty$ in any case. In fact, it can be seen from (15), (16), (19), and (20) that $\left(x_{i} / y_{i}\right)-1, i=1,2$, approach zero at the same rate as $t \rightarrow \infty$, as do $\left(x_{i} / y_{i}\right)^{\prime}, i=1$, 2. We also note that the results of these four equations can be written as

$$
x_{i} / y_{i}=1+O\left(\sigma y_{1} / y_{2}\right) \quad \text { and } \quad\left(x_{i} / y_{i}\right)^{\prime}=O\left(\sigma / r y_{2}^{2}\right), \quad i=1,2 .
$$




\section{MAIN RESUlts}

Theorem 2. Suppose that $\int^{\infty}(f-g) y_{2}^{2} d t$ converges. Let $I$ and $\sigma$ be as in (11) and (12). Then (11) has a solution $x_{1}$ that satisfies (15) and (16), and a solution $x_{2}$ such that

$$
\frac{x_{2}-y_{2}}{y_{1}}=O(\sigma)
$$

and

$$
\left(\frac{x_{2}-y_{2}}{y_{1}}\right)^{\prime}=O\left(\frac{\sigma \rho^{\prime}}{\rho}\right) .
$$

Proof. We have already proved the assertion concerning $x_{1}$. For the assertion concerning $x_{2}$, we use the contraction mapping theorem. If

$$
x_{2}(t)=y_{2}(t)+\int_{t}^{\infty}\left(y_{2}(s) y_{1}(t)-y_{1}(s) y_{2}(t)\right)(f(s)-g(s)) x_{2}(s) d s,
$$

then $x_{2}$ satisfies (1). Although this suggests a transformation to work with, it is better to use a transformation with the fixed point $\zeta$, where

$$
\zeta=\left(x_{2}-y_{2}\right) / y_{1} \text {. }
$$

Rewriting (223) in terms of $\zeta$ yields

$$
\begin{aligned}
\zeta(t)= & \int_{t}^{\infty}\left(y_{2}(s)-y_{1}(s) \rho(t)\right)(f(s)-g(s)) y_{2}(s) d s \\
& +\int_{t}^{\infty}\left(y_{2}(s)-y_{1}(s) \rho(t)\right)(f(s)-g(s)) y_{1}(s) \zeta(s) d s .
\end{aligned}
$$

We use the transformation $\mathcal{T} z=Q+\mathcal{L} z$, where

$$
Q(t)=\int_{t}^{\infty}\left(y_{2}(s)-y_{1}(s) \rho(t)\right)(f(s)-g(s)) y_{2}(s) d s
$$

and

$$
(\mathcal{L} z)(t)=\int_{t}^{\infty}\left(y_{2}(s)-y_{1}(s) \rho(t)\right)(f(s)-g(s)) y_{1}(s) z(s) d s .
$$

From (10), (11), and (13),

$$
Q(t)=I(t)-\rho(t) H(t)=-\rho(t) \int_{t}^{\infty} I(1 / \rho)^{\prime} d s,
$$

so $|Q(t)| \leq \sigma(t)$, from (12). Moreover,

$$
Q^{\prime}=I^{\prime}-\rho H^{\prime}-H \rho^{\prime}=-H \rho^{\prime}
$$

so

$$
\left|Q^{\prime}(t)\right| \leq 2 \sigma(t) \rho^{\prime}(t) / \rho(t),
$$

from (14). Therefore we let $\mathcal{T}$ act on the Banach space $\mathcal{B}$ of functions $z$ on $\left[t_{0}, \infty\right)$ such that

$$
z=O(\sigma) \quad \text { and } \quad z^{\prime}=O\left(\sigma \rho^{\prime} / \rho\right)
$$

with norm

$$
\|z\|=\sup _{t \geq t_{0}}\left\{\max \left\{\frac{|z|}{\sigma}, \frac{\rho\left|z^{\prime}\right|}{\sigma \rho^{\prime}}\right\}\right\} .
$$


We will show that $\mathcal{T}$ maps $\mathcal{B}$ into $\mathcal{B}$, and is a contraction if $t_{0}$ is sufficiently large. Since $Q \in \mathcal{B}$, it suffices to show that $\mathcal{L}$ is a contraction of $\mathcal{B}$ if $t_{0}$ is sufficiently large. To this end, suppose $z \in \mathcal{B}$ and $t_{0} \leq t<T$, and consider the finite integral

$$
w_{T}(t ; z)=\int_{t}^{T}\left(y_{2}(s)-y_{1}(s) \rho(t)\right)(f(s)-g(s)) y_{1}(s) z(s) d s .
$$

From (15) and (8),

$$
\begin{aligned}
w_{T}(t ; z)= & -\int_{t}^{T}(\rho(s)-\rho(t)) z(s) G^{\prime}(s) d s \\
= & -(\rho(T)-\rho(t)) z(T) G(T) \\
& +\int_{t}^{T}(\rho(s)-\rho(t)) G(s) z^{\prime}(s) d s \\
& +\int_{t}^{T} z(s) G(s) \rho^{\prime}(s) d s .
\end{aligned}
$$

From (14) and (24),

$$
\begin{gathered}
|(\rho(T)-\rho(t)) z(T) G(T)|<2\|z\| \sigma^{2}(T) / \rho(T) \rightarrow 0 \text { as } T \rightarrow \infty, \\
\left|(\rho(s)-\rho(t)) G(s) z^{\prime}(s)\right| \leq 2\|z\| \sigma^{2}(s) \rho^{\prime}(s) / \rho^{2}(s), \quad s \geq t,
\end{gathered}
$$

and

$$
\left|z(s) G(s) \rho^{\prime}(s)\right| \leq 2\|z\| \sigma^{2}(s) \rho^{\prime}(s) / \rho^{2}(s) .
$$

Therefore we can let $T \rightarrow \infty$ in (25) and conclude that

$$
(\mathcal{L} z)(t)=-\int_{t}^{\infty}(\rho(s)-\rho(t)) z(s) G^{\prime}(s) d s
$$

exists and satisfies the inequality

$$
|(\mathcal{L} z)(t)|<4\|z\| \int_{t}^{\infty} \frac{\sigma^{2} \rho^{\prime}}{\rho^{2}} d s<4\|z\| \frac{\sigma^{2}(t)}{\rho(t)} .
$$

From (26),

$$
(\mathcal{L} z)^{\prime}(t)=\rho^{\prime}(t) \int_{t}^{\infty} z G^{\prime} d s=-\rho^{\prime}(t)\left(z(t) G(t)+\int_{t}^{\infty} G z^{\prime} d s\right) .
$$

From (14) and (24), the last integral converges absolutely and

$$
\left|(\mathcal{L} z)^{\prime}(t)\right| \leq 2\|z\| \rho^{\prime}(t)\left(\frac{\sigma^{2}(t)}{\rho^{2}(t)}+\int_{t}^{\infty} \frac{\sigma^{2} \rho^{\prime}}{\rho^{3}} d s\right)<4\|z\| \frac{\sigma^{2}(t) \rho^{\prime}(t)}{\rho^{2}(t)} .
$$

From this and (27),

$$
\|(\mathcal{L} z)\|<4\|z\| \sigma(t) / \rho(t) .
$$

Hence $\mathcal{L}$ (and consequently $\mathcal{T}$ ) is a contraction of $\mathcal{B}$ if $\sigma\left(t_{0}\right) / \rho\left(t_{0}\right)<1 / 4$. Therefore there is a (unique) $\zeta \in \mathcal{B}$ such that $\mathcal{T} \zeta=\zeta$, and the function $x_{2}$ defined by $x_{2}=y_{2}+y_{1} \zeta\left(t \geq t_{0}\right)$ is a solution of (1) that satisfies (21) and (22). We can extend the definition of $x_{2}$ back to $t=a$.

Corollary 1. Under the assumptions of Theorem 园, $x_{2}$ satisfies (19) and (20). 
Proof. Since $y_{2} / y_{1}=\rho$, (21) implies that $y_{2}$ satisfies (19) and

$$
x_{2} / y_{1}=\rho+O(\sigma) .
$$

From (22),

$$
\left(x_{2} / y_{1}\right)^{\prime}=\rho^{\prime}(1+O(\sigma / \rho)) \text {. }
$$

Therefore

$$
\begin{aligned}
\left(\frac{x_{2}}{y_{2}}\right)^{\prime} & =\left(\frac{x_{2}}{y_{1} \rho}\right)^{\prime}=\left(\frac{x_{2}}{y_{1}}\right)^{\prime} \frac{1}{\rho}-\frac{x_{2}}{y_{1}} \frac{\rho^{\prime}}{\rho^{2}} \\
& =\frac{\rho^{\prime}}{\rho}(1+O(\sigma / \rho))-\frac{\rho^{\prime}}{\rho^{2}}(\rho+O(\sigma))=O\left(\frac{\sigma \rho^{\prime}}{\rho^{2}}\right) .
\end{aligned}
$$

It is natural to ask whether the convergence of $\int^{\infty}(f-g) y_{2}^{2} d t$ is necessary for the existence of a solution $x_{2}$ of (1) such that

$$
x_{2}=y_{2}(1+o(1 / \rho)) \quad \text { and } \quad\left(x_{2} / y_{2}\right)^{\prime}=o\left(\rho^{\prime} / \rho^{2}\right) .
$$

Although we do not know the answer to this question, we offer the following related theorem.

Theorem 3. If (1) has a solution $x_{2}$ that satisfies (19) and (20) for some positive monotonic function $\sigma$ such that $\lim _{t \rightarrow \infty} \sigma(t)=0$, then

$$
\int_{t}^{\infty}(f-g) y_{1} y_{2} d t=O(\sigma / \rho) \text {. }
$$

Moreover, if

$$
\int^{\infty} \frac{\sigma \rho^{\prime}}{\rho} d t<\infty
$$

then $\int^{\infty}(f-g) y_{2}^{2} d t$ converges.

Proof. From (20), $R(t)=\int_{t}^{\infty}\left(x_{2} / y_{2}\right)^{\prime} d t$ converges absolutely and

$$
R=O(\sigma / \rho) \text {. }
$$

If $t>T$, define

$$
R_{T}(t)=\int_{t}^{T}\left(\frac{x_{2}}{y_{2}}\right)^{\prime} d s
$$

From (5) and (6),

$$
\left(\frac{x_{2}}{y_{2}}\right)^{\prime}=\frac{y_{2} x_{2}^{\prime}-x_{2} y_{2}^{\prime}}{y_{2}^{2}}=u \frac{\rho^{\prime}}{\rho^{2}}
$$

where

$$
u=r\left(y_{2} x_{2}^{\prime}-x_{2} y_{2}^{\prime}\right) .
$$

From (11) and (2),

$$
u^{\prime}=(f-g) y_{2} x_{2} .
$$

Therefore

$$
R_{T}(t)=\frac{u(t)}{\rho(t)}-\frac{u(T)}{\rho(T)}+\int_{t}^{T}(f-g) y_{1} x_{2} d s .
$$


From (20) and (31), $u=o(\sigma)$, so we can let $T \rightarrow \infty$ and invoke (30) to conclude that

$$
\hat{R}(t) \stackrel{\mathrm{df}}{=} \int_{t}^{\infty}(f-g) y_{1} x_{2} d s=O(\sigma / \rho) .
$$

Now let

$$
\begin{aligned}
S_{T}(t) & =\int_{t}^{T}(f-g) y_{1} y_{2} d s=-\int_{t}^{T} \frac{y_{2}}{x_{2}} \hat{R}^{\prime} d s \\
& =\frac{y_{2}(t)}{x_{2}(t)} \hat{R}(t)-\frac{y_{2}(T)}{x_{2}(T)} \hat{R}(T)+\int_{t}^{T} \hat{R}\left(\frac{y_{2}}{x_{2}}\right)^{\prime} d s
\end{aligned}
$$

But

$$
\left(\frac{y_{2}}{x_{2}}\right)^{\prime}=-\frac{y_{2}^{2}}{x_{2}^{2}}\left(\frac{x_{2}}{y_{2}}\right)^{\prime}=O\left(\frac{\sigma \rho^{\prime}}{\rho^{2}}\right)
$$

from (19) and (20). From this and (32), we can let $T \rightarrow \infty$ in (33) to conclude that

$$
S(t) \stackrel{\mathrm{df}}{=} \int_{t}^{\infty}(f-g) y_{1} y_{2}=O(\sigma / \rho) .
$$

This verifies (28). If (29) holds and $T>a$, then

$$
\int_{a}^{T}(f-g) y_{2}^{2} d t=-\int_{a}^{T} \rho S^{\prime} d t=\rho(a) S(a)-\rho(T) S(T)+\int_{a}^{T} S \rho^{\prime} d t .
$$

Since (34) implies that $\lim _{T \rightarrow \infty} \rho(T) S(T)=0$ and (29) and (34) together imply that $\int^{\infty} S \rho^{\prime} d t$ converges, (35) implies that $\int^{\infty}(f-g) y_{2}^{2} d t$ converges.

\section{EXAMPLES}

Examples illustrating our results can be constructed by letting

$$
g(t)=f(t)+\frac{u(t) S(t)}{y_{2}^{2}(t)}, \quad t \geq a,
$$

where $u$ and $S$ are continuously differentiable and $S$ has a bounded antiderivative $C$ on $[a, \infty)$, while $\lim _{t \rightarrow \infty} u(t)=0$ and $\int^{\infty}\left|u^{\prime}(t)\right| d t<\infty$. Then

$$
\int_{t}^{\infty}(f(s)-g(s)) y_{2}^{2}(s) d s=-\int_{t}^{\infty} u(s) S(s) d s=-\left.u(s) C(s)\right|_{t} ^{\infty}+\int_{t}^{\infty} u^{\prime}(s) C(s) d s
$$

converges, and the convergence may be conditional. Here we may take

$$
\sigma(t)=M \sup _{\tau \geq t}\left(|u(\tau)|+\int_{\tau}^{\infty}\left|u^{\prime}(s)\right| d s\right),
$$

where $M$ is an upper bound for $C$ on $[a, \infty)$.

For a specific example, consider the equation

$$
x^{\prime \prime}+\frac{\sin t}{t^{2}(\log t)^{\alpha}} x=0, \quad t \geq a>0 \quad(\alpha>0),
$$

as a perturbation of $y^{\prime \prime}=0$. Our results imply that (36) has solutions $x_{1}$ and $x_{2}$ such that

$$
x_{1}(t)=1+O\left(t^{-1}(\log t)^{-\alpha}\right), \quad x_{1}^{\prime}(t)=O\left(t^{-2}(\log t)^{-\alpha}\right)
$$

and

$$
x_{2}(t)=t+O\left((\log t)^{-\alpha}\right), \quad x_{2}^{\prime}(t)=1+O\left(t^{-1}(\log t)^{-\alpha}\right) .
$$




\section{REFERENCES}

[1] S. Chen, Asymptotic integration of nonoscillatory second order differential equations, Trans. Amer. Math. Soc. 327 (1991), 853-866. MR 92a:34057

[2] N. Chernyavskaya and L. Shuster, Necessary and sufficient conditions for the solvability of a problem of Hartman and Wintner, Proc. Amer. Math. Soc. 125 (1997), 3213-3228. MR 98f:34045

[3] N. Chernyavskaya, On a problem of Hartman and Wintner, Proc. Roy. Soc. Edinburgh Sect A128 (1998), 1007-1022. MR 99h:34077

[4] P. Hartman, Ordinary Differential Equations, Wiley, New York, 1964. MR 30:1270

[5] J. Šimša, Asymptotic integration of a second order ordinary differential equation, Proc. Amer. Math. Soc. 101 (1987), 96-100. MR 89b:34129

[6] W. F. Trench, Linear perturbations of a nonoscillatory second order equation, Proc. Amer. Math. Soc. 97 (1986), 423-428. MR 87g:34036

95 Pine Lane, Woodland Park, Colorado 80863

E-mail address: wtrench@trinity.edu 